\title{
Modulus Search for Elliptic Curve Cryptosystems
}

\author{
Kenji Koyama, Yukio Tsuruoka, and Noboru Kunihiro \\ NTT Communication Science Laboratories \\ 2-4, Hikaridai, Seika-cho, Soraku-gun, Kyoto, 619-0237 Japan \\ \{koyama, tsuru, kunihiro\}@cslab.kecl.ntt.co.jp
}

\begin{abstract}
We propose a mathematical problem, and show how to solve it elegantly. This problem is related with elliptic curve cryptosystems (ECC). The solving methods can be applied to a new paradigm of key generations of the ECC.
\end{abstract}

\section{Problem}

Celebrating Asiacrypt'99 held in November (11th month) of 1999, we propose a mathematical problem after these numbers.

Let $c, x, y$ be integers such that $0 \leq x<c$ and $0 \leq y<c$.
Define $N(c)$ be the number of points $(x, y)$ satisfying
$\quad y^{2} \equiv x^{3}+11 x(\bmod c) \quad(1)$
Obtain all values of $c$ such that $N(c)=1999$.

\section{Solving the Problem}

\subsection{Observing the Behaviour of $N(c)$}

This problem itself is easy to understand for junior highschool students, however, solving it may be a little difficult for them. It would be moderate for modern cryptographers.

First, observe the behaivior of $N(c)$ concerning equation (1) from small numerical examples. When $c=7$, the integer points $(x, y)$ of equation (1) are $(0,0),(2,3),(2,4),(3,2),(3,5),(6,3),(6,4)$. Thus, we have $N(7)=7$. Similarly, we compute the values of $N(c)$ for integers $c$ such that $1 \leq c \leq 18$, and primes below 100. The result is shown in Table 1.

Table 1. Examples of $N(c)$

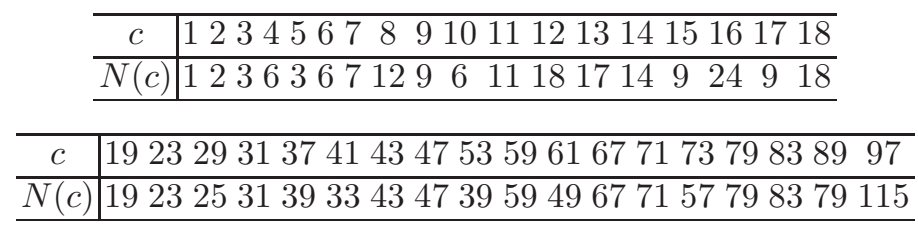

We can find the properties of $N(c)$ if we observe Table 1 carefully. 


\subsection{Obtaining one Solution}

Hereafter, considering equation (1) as general as possible, we can obtain the following theorems for the properties of $N(c)$.

Theorem A: Define $N_{u}(c)$ be the number of points for a general congruence:

$$
f(x, y) \equiv 0(\bmod c)
$$

If $c$ is composite (i.e. not prime), then $N_{u}(c)$ is composite. In particular, when $c_{1}$ and $c_{2}$ are coprime, we have

$$
N_{u}\left(c_{1} c_{2}\right)=N_{u}\left(c_{1}\right) N_{u}\left(c_{2}\right) .
$$

Of course, Theorem A holds for $N(c)$ concerning equation (1). If $c$ is a prime power, we have Theorem B.

Theorem B : Define $N_{s}(c)$ be the number of points for congruence:

$$
y^{2} \equiv x^{3}+a x(\bmod c)
$$

Let $p$ be a prime and $c=p^{n}(n \geq 2)$.

(i) If $p(\neq 2)$ is coprime to a, then $N_{s}\left(p^{n}\right)=p^{n-1} \cdot N_{s}(p)$,

(ii) If $p(\neq 2)$ divides $a$, then $N_{s}\left(p^{n}\right)=(2 p-1) \cdot N_{s}(p)^{n-1}$.

(iii) $N_{s}(2)=2$. When $a=11, N_{s}\left(2^{n}\right)=3 \cdot 2^{n-1}$.

Putting $a=11$ in equation (4), Theorem B holds for $N(c)$ concerning equation (1).

Theorem C: Define $N_{s}(c)$ in the same way as Theorem B. If $p$ is a prime such that $p \equiv 3(\bmod 4)$ and $a$ is coprime to $p$, then $N_{s}(p)=p$.

Theorem $\mathrm{C}$ holds for $N(c)$ concerning equation (1).

In the problem, we just said on purpose " $c$ is an integer." I did not say " $c$ is restricted to a prime." Theorem A can be rewritten as "If $N(c)$ is a prime, then $c$ is a prime." Note that 1999 is a prime. We can observe that $c$ is a prime because $N(c)=1999$. Moreover, noticing $1999 \equiv 3(\bmod 4)$, we can find from Theorem $\mathrm{C}$ that $N(1999)=1999$. That is, a prime $c$ satisfying $N(c)=1999$ and $c \equiv 3(\bmod 4)$ is only 1999 .

\subsection{Obtaining other Solutions}

In the problem, we said "Obtain all values of $c$. " Therefore, the remaining candidates of $c$ must be primes with $c \equiv 1(\bmod 4)$. What is the range for searching the remaining prime candidates? We show here a strong theorem, which is called Hasse's Theorem and popular in elliptic curve theory.

Hasse's Theorem: Let $p$ be a prime and coprime to $4 a^{3}+27 b^{2}$. Consider an elliptic curve over prime field $G F(p)$ :

$$
y^{2} \equiv x^{3}+a x+b(\bmod p) .
$$


Excluding a point at infinity, the number of points on this curve, denoted by $N_{w}(p)$, is given by in the following range:

$$
p-2 \sqrt{p} \leq N_{w}(p) \leq p+2 \sqrt{p} .
$$

If $4 a^{3}+27 b^{2}$ is coprime to $p$, the curve of equation (5) becomes an elliptic curve, which is a cubic curve without singular points. Equation (5) is called Weierstraß form. Putting $a=11, b=0$, equation (5) becomes equation (1). The number of points on elliptic curves is usually called an order, including one point at infinity. Thus, the order is expressed as $N_{w}(p)+1$. In the problem, to avoid difficulty of understanding of a point at infinity, we define $N(c)\left(, N_{u}(c), N_{s}(c)\right.$, and $\left.N_{w}(c)\right)$ excluding a point at infinity. Even for researchers familiar with elliptic curves and their orders, the proposed problem must be a new application paradigm, in which a modulus is determined from given an order of elliptic curve.

Naive Method: Method 1 First, to restrict the range of the solutions $c$ of the problem, we need to get a lemma of Hasse's theorem. Given $N_{w}(p)(=N(p))$, a prime modulus $p$ of elliptic curve is restricted between a certain range. This range is obtained from equation (5). By rewriting equation (5), we have

$$
p^{2}-2\left(N_{w}(p)+2\right) p+N_{w}(p)^{2} \leq 0 .
$$

By solving $p$ for this quadratic form, we have an inequality:

$$
N_{w}(p)+2-2 \sqrt{N_{w}(p)+1} \leq p \leq N_{w}(p)+2+2 \sqrt{N_{w}(p)+1}
$$

Putting $N(p)=N_{w}(p)=1999$, we can get an explicit range as $1911.6 \leq p \leq$ 2090.4. Thus, the values of modulus $c$ should be searched from 1912 to 2090 . In this range, there are twelve primes $(\equiv 1(\bmod 4))$ as $1913,1933,1949,1973$, 1993, 1997, 2017, 2029, 2053, 2069, 2081 and 2089. The most naive method is to compute $N(p)$ for all of these twelve values of $p$, and check whether $N(p)=1999$. We can find that only $p=2017$ satisfies $N(p)=1999$.

Elegant Method: Method 2 Elegant methods can be constructed by decreasing the number of candidates of modulus by a simple analysis. Note that for a prime with $p \equiv 1(\bmod 4)$, there are integers $U, V$ ( $U$ is odd and $V$ is even)such that

$$
p=U^{2}+V^{2} \text {. }
$$

The values of $(U, V)$ is uniquely determined and easily obtained. In elliptic curve theory, the following theorem $\mathrm{D}$ is known,

Theorem $\mathbf{D}:$ Let $p$ be a prime satisfying $p \equiv 1(\bmod 4)$ and $p=U^{2}+V^{2}$. If $a(\neq 0)$ is coprime to $p$, the number of points of equation (4), denoted by $N_{s}(p)$, is one of the following four candidates:

$$
N_{s}(p)=p \pm 2 U, \quad p \pm 2 V
$$


Let $U^{\prime}=|p-1999| / 2$ and $W=p-U^{\prime 2}$. Observing theorem $\mathrm{D}, W$ must be a square to satisfy $N(p)=1999$ for a prime with $p \equiv 1(\bmod 4)$. For each $p$ of twelve candidates, the computed values of $U^{\prime}$ and $W$ are shown in Table 2 . Observing Table 2, only four primes such that $p=1913,2017,2081$ and 2089 imply that $W$ are squares. For these reduced four candidates $p$, the computed values of $N(p)$ are also shown in Table 2 . We can find that only $p=2017$ satisfies $N(p)=1999$.

Table 2. Reduction of primes $p$ and reduced $N(p)$

\begin{tabular}{|c||c|c|c|c|c|c|c|c|c|c|c|c|}
\hline$p$ & 1913 & 1933 & 1949 & 1973 & 1993 & 1997 & 2017 & 2029 & 2053 & 2069 & 2081 & 2089 \\
\hline$U^{\prime}$ & 43 & 33 & 25 & 13 & 3 & 1 & 9 & 15 & 27 & 35 & 41 & 45 \\
\hline$W$ & 64 & 844 & 1324 & 1804 & 1984 & 1996 & 1936 & 1804 & 1324 & 844 & 400 & 64 \\
\hline$N(p)$ & 1929 & - & - & - & - & - & 1999 & - & - & - & 2121 & 2105 \\
\hline
\end{tabular}

More Elegant Method: Method 3 We would show more elegant and efficient method. If we apply equation (8) and theorem D extendedly, we do not need to know and use Hasse's Theorem and its lemma directly. Note that for prime $p$ with $p \equiv 1(\bmod 4), N_{s}(p)+1$ is represented as one of four values:

$$
N_{s}(p)+1=(U \pm 1)^{2}+V^{2}, \quad U^{2}+(V \pm 1)^{2}
$$

Thus, if given $N_{s}(p)+1$ is represented as a sum of two squares as $N_{s}(p)+1=$ $\alpha^{2}+\beta^{2}(\alpha \leq \beta)$ then $(U, V)$ is one of $(\alpha \pm 1, \beta)$ and $(\alpha, \beta \pm 1)$. We compute candidates of $p$ from these candidates of $(U, V)$. Then we do primality test for $p$ and check whether $N(p)=1999$. The passed $p$ become solutions.

We show the above method concretely. Since $N_{s}(p)+1=1999+1=2000$, we search $(\alpha, \beta)$ such that $2000=\alpha^{2}+\beta^{2}$, noticing $\alpha \leq \sqrt{2000 / 2}$. We obtain two pairs $(\alpha, \beta)=(8,44),(20,40)$. ¿From each pair, eight candidates of $p_{i}(1 \leq i \leq 8)$ can be computed as

$$
\begin{aligned}
& p_{1}=(8+1)^{2}+44^{2}=2017, p_{2}=(8-1)^{2}+44^{2}=1985, \\
& p_{3}=8^{2}+(44+1)^{2}=2089, p_{4}=8^{2}+(44-1)^{2}=1913, \\
& p_{5}=(20+1)^{2}+40^{2}=2041, p_{6}=(20-1)^{2}+40^{2}=1981, \\
& p_{7}=20^{2}+(40+1)^{2}=2081, p_{8}=20^{2}+(40-1)^{2}=1921 .
\end{aligned}
$$

Among these values, only $p_{1}, p_{3}, p_{4}$ and $p_{7}$ are primes. These primes are congruent 1 modulo 4 , however, only $p_{1}=2017$ satisfies $N\left(p_{i}\right)=1999$. This method is more efficient than the method 2 because of less primality tests. It is interesting that four candidates derived by method 3 are the same as four candidates derived by method 2 .

Much more Elegant Method: Method 4 Moreover, much more elegant method can be constructed by observing the reduced candidates from another viewpoint. When $p \equiv 1(\bmod 4)$, order $S=N(p)+1$ is expressed by

$$
S=4 t+3+L(11, p)
$$


where $t$ is the number of the cases that $x^{3}+11 x$ become quadratic residues modulo $p$ when $1 \leq x \leq(p-1) / 2$. Generally, the Legendre symbol $L(d, p)$ means as follows. $L=1$ if $d(\neq 0)$ is a quadratic residue modulo prime $p$; $L=-1$ if $d(\neq 0)$ is a quadratic non-residue modulo prime $p ; L=0$ if $d=$ 0 . To satisfy $S=2000$, we need that $S \equiv 0(\bmod 4)$, and 11 is a quadratic residue modulo $p$. Among four primes 1913, 2017, 2081, and 2089, only $p=2017$ satisfies $L(11, p)=1$. Thus, we compute $N(p)$ for only $p=2017$, and we verify $N(2017)=1999$.

Note that method 2 and method 3 require four computations of $N(p)$, however, method 4 requires four computations of Legendre symbols and one computation of $N(p)$. Thus, method 4 is more efficient than methods 2 and 3 .

\section{Counting Points of the Curves}

\subsection{General Methods}

There are several ways to compute $N(c)$ from $c$. The most naive method is to count the points $(x, y)$ satisfying equation (1) by varying both $x$ and $y$ from 0 to $c-1$. The computational complexity is $O\left(c^{2}(\log c)^{2}\right)$. If $c$ is a prime, we can compute $N(c)$ using Legendre symbol $L$ as

$$
N(c)=\sum_{x=0}^{c-1}\left\{1+L\left(x^{3}+11 x, c\right)\right\} .
$$

We call this method the Legendre method. Since the Legendre symbol itself can be computed in $O\left((\log c)^{3}\right)$, computation of $N(c)$ by the Legendre method requires $O\left(c(\log c)^{3}\right)$. It is more efficient than the naive method.

When $c$ is about 4 digits, $N(c)$ can be computed in less than one second on a typical personal computer if the Legendre symbol method is used. When $c$ is about 200 digits, the computation of $N(c)$ is intractable even if the Legendre symbol method is used. For large $p$, counting the points (i.e. order or $N_{w}(p)$ ) on an elliptic curve over prime field $G F(p)$ had been a difficult problem historically. However, Schoof discovered an efficient method in 1985. The implementation is rather complicated, but it runs in polynomial time i.e. in $O\left((\log p)^{8}\right)$. Recently, an improved Schoof method, which is also called Schoof-Elkies-Atkin (SEA) method, is used and it runs in $O\left((\log p)^{6}\right)$. This newest counting method is used in the design of elliptic curve method (ECC). Note that ECC is a public-key cryptosystem, which is the most promising scheme in the next generation of the RSA scheme.

\subsection{Special Counting Method for the Problem}

Return to the problem. Since equation (1) has a restricted parameters $a, b$ and $p$, we can compute $N(p)$ analytically and efficiently using Theorem E. 
Theorem E : $N_{s}(p)$ is uniquely determined by

$$
N_{s}(p)=p-\overline{\left(\frac{-a}{\pi}\right)_{4}} \pi-\left(\frac{-a}{\pi}\right)_{4} \bar{\pi},
$$

where $p=\pi \bar{\pi}$, and $\pi$ is Gaussian integer $Z[i](i=\sqrt{-1})$, and $\pi \equiv 1(\bmod$ $2+2 i)$.

Note that $\left(\frac{-a}{\pi}\right)_{4}=\{1,-1, i,-i\}$, and computed as $\left(\frac{-a}{\pi}\right)_{4}=(-a)^{(p-1) / 4} \bmod \pi$.

Using Theorem E, we can easily compute $N(p)$ for each $p$. For example, when $p=2017$, we have $p=9^{2}+44^{2}$, and $N(2017)=2017-2 \times 9=1999$. The computational time on a typical computer using Theorem $\mathrm{E}$ is also less than one second.

\section{Solution}

The above discussion result in a solution of the problem. There are only $\mathrm{c}=1999$ and 2017 satisfying equation (1).

Note that if one try to search them on a computer without any knowledge or any analysis, it need infinite time. The theorems and discussions in this paper convince us that there are only two values of $c$ satisfying equation (1).

\section{Viewpoint of ECC Design}

In cryptographic design, there are two typical methods for constructing secure elliptic curves for the ECC: the SEA method (the point-counting method based on the improved Schoof algorithm) and the $\mathrm{CM}$ (Complex Multiplication) method. The SEA Method: The point-counting method computes an order of random curve modulo $p$ until the order satisfies the security. Given parameters $(a, b)$ and prime modulus $p$ of elliptic curve, the improved versions of Schoof algorithm can compute order $\# E(a, b, p)(=S)$ in $O\left((\log p)^{6}\right)$. Considering the time of the primality check of $p$ and the security check of $S$, including their success probability, the computational time for obtaining a suitable triple $(S,(a, b), p)$ based on the improved Schoof algorithm is $O\left((\log p)^{7}\right)$.

The CM method: The CM method chooses a secure order first from modulus $p$, then builds a curve with that order. Given the prime modulus $p$ of an elliptic curve, the Atkin-Morain algorithm and its variants compute the $j$-invariant of the curve, and obtains order $S$ and parameters $(a, b)$ satisfying $S=\# E(a, b, p)$. They run in $O\left((\log p)^{5}\right)$. Considering the time of the primality check of $p$ and the security check of $S$, including their success probability, the computational time for obtaining a suitable triple $(S,(a, b), p)$ based on the CM method is $O\left((\log p)^{6}\right)$.

That is, the CM method based on the Atkin-Morain algorithm is more efficient than the point-counting method based on the (improved) Schoof algorithm.

Now, we consider a new approach based on problem G and its solution, which follows. This approach, which we call the modulus-searching method, is 
in another direction among $(S,(a, b), p)$, and is different from SEA method and CM method.

Problem G: Given order $S$ and parameters $(a, b)$ of an elliptic curve, construct an efficient algorithm for determining a prime modulus $p$ satisfying $S=$ \#E( $(a, b, p)$, if such $p$ exists.

When the values of $a, b$ and $S$ are arbitrary, we can construct a general algorithm for problem $\mathrm{G}$. We can find a prime $p$ satisfying $S=\# E(a, b, p)$ if $p$ exists. The time complexity of this algorithm is $O\left(\sqrt{S}(\log S)^{2}\right)$. This general but simple algorithm is not efficient for large $S$ because there are many candidates for the prime modulus.

Therefore, we focus on the constructions of restricted elliptic curves $E(a, b, p)$ with $\{a \neq 0, b=0\}$, whose $j$-invariant is 1728 , and $\{a=0, b \neq 0\}$, whose $j$ invariant is 0 . Note that the Atkin-Morain algorithm excludes these "simple" curves. There are a few studies on the ECC using such curves. If $\{a \neq 0, b=0$ and $p \equiv 1(\bmod 4)\}$ or $\{a=0, b \neq 0$ and $p \equiv 1(\bmod 3)\}$, then orders of such curves can be easily computed in $O\left((\log p)^{3}\right)$ by the point-counting method based on complex multiplications over the imaginary quadratic field $\mathbf{Q}(\sqrt{-1})$ or $\mathbf{Q}(\sqrt{-3})$. This "special point-counting algorithm" is faster than the general (improved) Schoof algorithm. This "special point-counting method" constructs a suitable triple $(S,(a, b), p)$ in $O\left((\log p)^{5}\right)$. ¿From the viewpoint of problem 1 , however, there have been no concrete proposals or deep discussions of efficient algorithms.

In [1] we proposed efficient algorithms for determining prime modulus $p$ from given order $S$ and parameters $(a, b)$ of elliptic curve $E(a, b, p): y^{2} \equiv x^{3}+a x+$ $b(\bmod p)$, where $\{a \neq 0, b=0\}$ or $\{a=0, b \neq 0\}$. First we choose secure order $S$ from its size. Next we search prime modulus $p$ satisfying $S=\# E(a, b, p)$. We can obtain a suitable triple $(S,(a, b), p)$ in polynomial time $O\left((\log S)^{5}\right)$. The proposed approach is faster than the previous approaches based on the Schoof algorithm and the Atkin-Morain algorithm.

\section{References}

1. K. Koyama, N. Kunihiro and Y. Tsuruoka: "Modulus Searching Methods for Secure Elliptic Curve Cryptosystems", Proc. of 1999 Symposium on Cryptography and Information Security (SCIS 99), pp. 863-868 (1999). 7 